\title{
TU/e EmonONEN

\section{Simulations of droplet collisions with a diffuse interface model near the critical point}

\section{Citation for published version (APA):}

Gelissen, E. J., van der Geld, C. W. M., Kuipers, J. A. M., \& Kuerten, J. G. M. (2018). Simulations of droplet collisions with a diffuse interface model near the critical point. International Journal of Multiphase Flow, 107, 208220. https://doi.org/10.1016/j.ijmultiphaseflow.2018.06.001

DOI:

10.1016/j.ijmultiphaseflow.2018.06.001

Document status and date:

Published: 01/10/2018

\section{Document Version:}

Accepted manuscript including changes made at the peer-review stage

\section{Please check the document version of this publication:}

- A submitted manuscript is the version of the article upon submission and before peer-review. There can be important differences between the submitted version and the official published version of record. People interested in the research are advised to contact the author for the final version of the publication, or visit the $\mathrm{DOI}$ to the publisher's website.

- The final author version and the galley proof are versions of the publication after peer review.

- The final published version features the final layout of the paper including the volume, issue and page numbers.

Link to publication

\section{General rights}

Copyright and moral rights for the publications made accessible in the public portal are retained by the authors and/or other copyright owners and it is a condition of accessing publications that users recognise and abide by the legal requirements associated with these rights.

- Users may download and print one copy of any publication from the public portal for the purpose of private study or research.

- You may not further distribute the material or use it for any profit-making activity or commercial gain

- You may freely distribute the URL identifying the publication in the public portal.

If the publication is distributed under the terms of Article 25fa of the Dutch Copyright Act, indicated by the "Taverne" license above, please follow below link for the End User Agreement:

www.tue.nl/taverne

Take down policy

If you believe that this document breaches copyright please contact us at:

openaccess@tue.nl

providing details and we will investigate your claim. 


\title{
Simulations of Droplet Collisions with a Diffuse Interface Model near the Critical Point
}

\author{
E.J. Gelissen ${ }^{1 *}$, C.W.M. van der Geld², J.A.M. Kuipers², J.G.M. Kuerten ${ }^{1}$ \\ ${ }^{1}$ Department of Mechanical Engineering \\ ${ }^{2}$ Department of Chemical Engineering and Chemistry \\ Eindhoven University of Technology
}

June 20, 2018

\begin{abstract}
In the present study a method is presented for simulations of droplet collisions in three spatial dimensions under non-isothermal conditions using a Diffuse Interface Model, which is based on the non-isothermal Navier-Stokes-Korteweg equations combined with the Van der Waals equation of state. This particular Diffuse Interface Model can only be used for a single component fluid and the temperature needs to be close to the critical point of the fluid to prevent the interfacial thickness from being too small compared to the size of the computational domain. Results are produced with a numerical method based on the finitevolume approach in combination with a Total Variation Diminishing time-integration scheme. Simulations of droplet collisions show the existence of multiple collision regimes for which the energy transfer and dissipation processes during the collisions are studied in detail.
\end{abstract}

Keywords: Droplet Collisions, Diffuse Interface Model, Navier-Stokes-Korteweg equations, Van der Waals equation

\section{Introduction}

Collision of liquid droplets is a complicated phenomenon in many industrial and geophysical processes, including raindrop formation, liquid-liquid extraction, spray coatings and spray drying processes. In internal combustion engines the development of the fuel injection spray, and hence the combustion process, is significantly influenced by droplet collisions. Understanding the mechanism of droplet collisions is therefore of great importance for optimizing the combustion process to achieve better efficiency and lower emissions.

Outcomes of droplet collisions can be categorized into four different types: bouncing, coalescence, separation, and shattering collisions. In bouncing collisions, contact of droplet surfaces is prevented by a separating gas film. Coalescence collisions refer to collisions in which two droplets permanently combine and generate one single droplet. Separation collision occurs when two droplets combine temporarily and later separate, possibly in more than two droplets. Shattering collisions are characteristic for high relative velocity collisions where the colliding droplets disintegrate into a cluster of many smaller droplets. Furthermore, there are many subcategories of droplet collisions within the categories generalized above.

The type of outcome of a droplet collision is determined by multiple parameters such as the kinetic energy of the collision, the diameter of the droplets and fluid properties like surface tension and viscosity. In the present study only collisions with equally sized droplets are considered. A convenient way of characterizing droplet collisions is by means of dimensionless numbers. Two dimensionless numbers suffice to characterize collisions of equally sized droplets: the collision Weber number $W e_{c}$ and the impact parameter $\mathscr{X}$.

$$
W e_{c}=\frac{\rho_{l}\left|\vec{v}_{r e l}\right|^{2} D}{\sigma}, \quad \mathscr{X}=\left(1-\left(\frac{\vec{v}_{r e l}}{\left|\vec{v}_{r e l}\right|} \cdot \frac{\vec{x}_{r e l}}{\left|\vec{x}_{r e l}\right|}\right)^{2}\right)^{\frac{1}{2}}
$$

*Corresponding author

E-mail adress: e.j.gelissen@tue.nl

Tel.: +31402472877

Postal adress: P.O. Box 513, 5600 MB Eindhoven, the Netherlands 
with $\rho_{l}$ the liquid mass density, $\sigma$ the surface tension coefficient, $\vec{v}_{r e l}$ the relative velocity vector of the colliding droplets, $D$ the droplet diameter and $\vec{x}_{r e l}$ the relative position vector between the centers of the colliding droplets (Figure 1, $\left|\vec{x}_{r e l}\right|=D$ for equal sized droplets). The collision Weber number represents the ratio between inertial forces and the surface tension coefficient, whereas the impact parameter gives a measure of the degree to which the collision is head-on or grazing and has a value between zero and unity.

Experimental investigations of droplet collisions have been performed by numerous authors, including Ashgriz and Poo [3], Qian and Law [30], Estrade et al. [12], Willis and Orme [46], Brenn and Kolobaric [4] and Gotaas et al. [14]. The experimental results are usually presented in a collision regime map with the collision Weber number on the horizontal axis, the impact parameter on the vertical axis and the boundaries between the collision outcomes indicated. Figure 2 shows a sketch of a typical collision regime map of water droplets in air, with three different regimes that are typically observed: coalescence, reflexive separation and stretching separation. Bouncing and shattering collisions are usually not observed for collisions of water droplets in air at atmospheric pressure. Coalescence is observed for low Weber number collisions at all impact parameters. Reflexive separation occurs for (near) head-on collisions at higher Weber numbers, while stretching separation occurs for large impact parameter collisions at higher Weber numbers. To describe the boundaries between the collision outcomes, many correlations have been proposed. An overview of the available correlations can be found in Krishnan and Loth [17]. However, as far as the authors know, no experimental results of droplet collisions with the fluid near the critical point have ever been published.

There is a large amount of disparity between droplet collision regime maps that were published in literature. The shape of the regime map depends heavily on the ratio between the surface tension coefficient and the viscosity of the fluid, which is specific for each fluid considered. Additionally, the properties of the gas/vapor surrounding the droplets has a large influence on whether droplet bouncing is observed in the regime map. For example: Willis and Orme [46] performed experiments with droplet collisions in a near vacuum environment and found no bouncing in the regime map. Qian and Law performed experiments with water and hydrocarbon droplets in air at $1 \mathrm{~atm}$. of pressure. Under these conditions droplet bouncing was only observed with hydrocarbon droplets and not with water droplets. Increasing the air pressure to $2.7 \mathrm{~atm}$. led to droplet bouncing also being observed with water droplets.

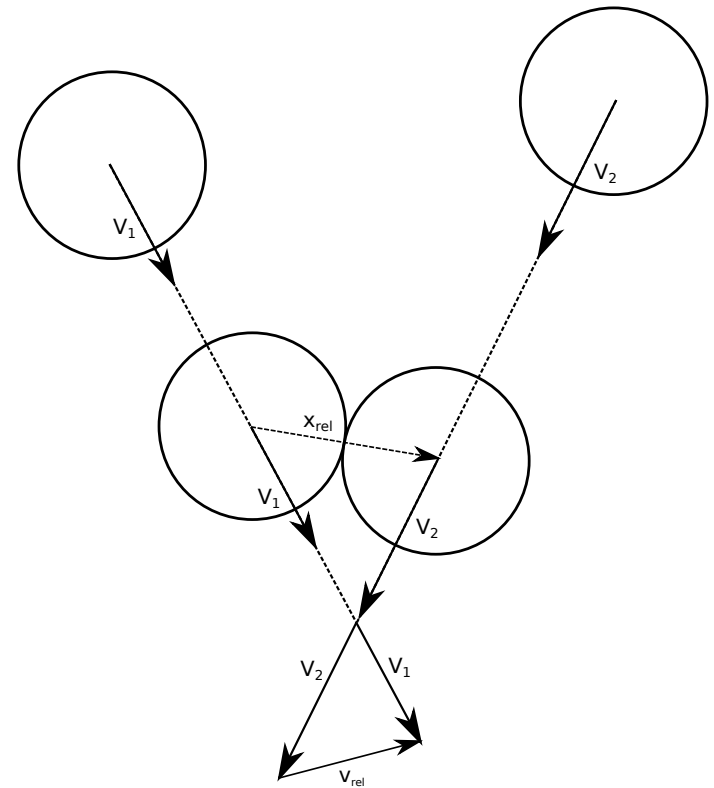

Figure 1: Droplet collision of equal sized droplets with $\vec{v}_{r e l}$ the relative velocity vector and $\vec{x}_{r e l}$ the relative position vector.

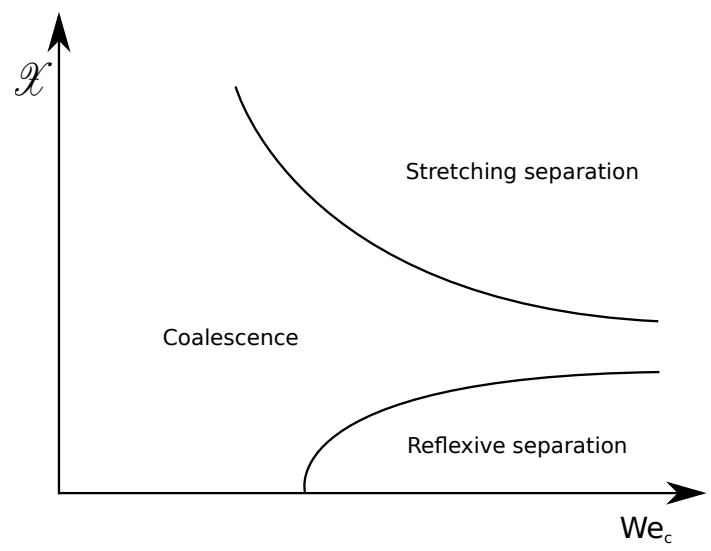

Figure 2: Typical collision regime map of water droplets in air [30].

Numerical simulations of droplet collisions involve simulating the movement of an interface separating two different fluid phases in a computational domain. The use of fixed grid simulation methods is more suitable when the deformation/movement of the interface is large, since the alternative moving grid methods requires frequent regeneration of the grid to accommodate for the deformation/movement of the interface. Multiple methods have been developed for numerical simulations of moving interfaces on a fixed grid, which include the Volume of Fluid (VOF) method [15], the level-set method [1] and coupled level-set-VOF methods [42]. These methods account for the effects of surface tension by using a body force that acts on a narrow region which 
represents the interface. The VOF method uses a color function to capture the interface, whereas the level-set method captures the interface with the zero level curve of a continuous function. An alternative approach is the front-tracking method, which is based on the Lagrangian tracking of marker particles from which the interface can be reconstructed [35]. The front-tracking method prevents the regeneration of the fixed grid by introducing a second set of grid points, which evolve relative to the fixed grid. The key advantage of all of these methods is that only a single set of governing equations needs to be solved for the entire computational domain. However, these methods are not well adapted to describe topological changes of the interface, like coalescence and breakup phenomena. In the VOF method, coalescence happens automatically when two approaching interfaces share a common grid cell [13]. The Level-set method has similar problems: coalescence either happens automatically or it is not possible unless it is explicitly imposed [18]. In the front-tracking method, the coalescence process requires a specialized routine that relies on an empirical parameter called the film drainage time [31].

Alternatively, simulations of droplet collisions have also been done with the Lattice-Boltzmann Method (LBM) $[11,41]$ and the technique of Smoothed Particle Hydrodynamics (SPH) [23]. In both methods the behavior of the fluid is approximated by modeling the interaction of particles. In the LBM the discrete Boltzmann equation is solved on a discrete lattice. In SPH the movement and interaction of discrete particles is simulated and smoothing functions are used to reconstruct the field variables from the particle data.

The Diffuse Interface Method (DIM) differs from all the previously mentioned methods in that it resolves the interface region in a physically motivated manner. An interface between two phases will always have a finite thickness (even if only nanoscopic). The thickness of the interface is determined by the competition between molecular repulsion and molecular attraction. This competition can be modeled by introducing a free energy density function of the fluid which is dependent on both the mass density and the mass density gradients. This causes the thickness and behavior of the interface to be determined by intermolecular forces, represented by the free energy density function. A great advantage of DIM is that topological changes of the interface do not require any additional models or routines.

One can distinguish between two types of Diffuse Interface Methods, although both are based on an extended version of the Navier-Stokes equations. There are the Navier-Stokes-Cahn-Hilliard (NSCH) equations [34], which can be used for systems with immiscible liquids, and the Navier-Stokes-Korteweg (NSK) equations [29], which can be used for liquid-vapor flows of a single component. The DIM used in the present study is based on the NSK equations, which are combined with the Van der Waals equation of state. The capillary stresses at the liquid-vapor interface are accounted for by the Korteweg stress tensor, which depends on the mass density and its spatial derivatives.

With the Korteweg stress tensor in the Navier-Stokes equations, movement and topological changes of the liquid-vapor interface are accounted for in a physical way. The dynamics of the liquid-vapor interface is provided by the evolution of the mass density field. However, the inclusion of the capillary stress tensor does lead to dispersive behavior of the solution, since it contains a second-order spatial derivative of the mass density. Also, the isotherms of the Van der Waals equation contain a region of negative compressibility below the critical point of the fluid. This leads to a mixed hyperbolic-elliptic nature of the governing equations and therefore prevents the use of upwind based discretization methods, which are commonly used for compressible flow simulations.

Numerical simulations of liquid-vapor flows with DIM have previously been performed by Jamet et al. [16], Yue et al. [47], Onuki [25], Lamorgese and Mauri [19], Pecenko et al. [29], Desmarais and Kuerten [9], Liu et al. $[21,22]$ and Tian et al. [43]. Simulations of droplet collisions with DIM in two spatial dimensions were done by Yue et al. [47] and Pecenko et al. [29]. However, a droplet collision is a process that needs to be modeled in three spatial dimensions if a physically relevant result is to be obtained, since in two spatial dimensions no spherical droplets but rather two infinitely long cylinders are modeled. Additionally, the assumption of isothermal conditions is not recommended for liquid-vapor flows, since the characteristics of liquid-vapor flows are highly sensitive to spatial temperature variations and these variations are bound to occur due to viscous dissipation of kinetic energy. In the present study results are presented of numerical simulations of droplet collisions in three spatial dimensions under non-isothermal conditions. This combination makes these results unique in the sense that, to the best of the authors knowledge, similar results have not been published before.

The structure of the paper is the following. In section 2 the governing equations are introduced, accompanied by the underlying theoretical principles in the field of thermodynamics. Section 3 gives the spatial discretization method and the time integration scheme. Also, expressions required for the initialization of the simulations are given. In section 4 droplet collisions are studied near the critical point, together with a detailed analysis of the energy transfer and dissipation process. In section 5 the conclusions are summarized. 


\section{Governing equations}

\subsection{Navier-Stokes-Korteweg equations}

The system of governing equations for non-isothermal liquid-vapor flows consists of three conservation equations commonly referred to as the Navier-Stokes-Korteweg (NSK) equations. The NSK equations can be classified as a non-linear system of conservation laws, expressed in the conservation of mass, momentum and energy. In differential form they are:

$$
\begin{gathered}
\frac{\partial \tilde{\rho}}{\partial \tilde{t}}+\nabla \cdot(\tilde{\rho} \tilde{\boldsymbol{u}})=0 \\
\frac{\partial \tilde{\boldsymbol{\rho}} \tilde{u}}{\partial \tilde{t}}+\nabla \cdot(\tilde{\rho} \tilde{\boldsymbol{u}} \otimes \tilde{\boldsymbol{u}}+\tilde{P} \boldsymbol{I}-\tilde{\boldsymbol{\tau}}-\tilde{\boldsymbol{\xi}})=\mathbf{0} \\
\frac{\partial \tilde{\rho} \tilde{E}}{\partial \tilde{t}}+\nabla \cdot\left((\tilde{\rho} \tilde{E}+\tilde{P}) \tilde{\boldsymbol{u}}-(\tilde{\boldsymbol{\tau}}+\tilde{\boldsymbol{\xi}}) \cdot \tilde{\boldsymbol{u}}+\tilde{\boldsymbol{q}}+\tilde{\boldsymbol{j}_{E}}\right)=0
\end{gathered}
$$

with $\tilde{\boldsymbol{u}}$ the velocity vector, $\tilde{\boldsymbol{\tau}}$ the viscous stress tensor, $\tilde{\boldsymbol{\xi}}$ the capillary stress tensor, $\tilde{\boldsymbol{q}}$ the heat flux and $\tilde{\boldsymbol{j}_{E}}$ the interstitial working flux. The non-dimensional form of the governing equations is obtained by introducing scaling quantities. The scales for the mass density, temperature and pressure are the corresponding quantities at the critical point of the fluid: $\rho_{c}, T_{c}$ and $P_{c}$. The velocity and characteristic time scale are derived as follows:

$$
u_{0}=\sqrt{\frac{P_{c}}{\rho_{c}}} \quad t_{c}=\frac{L}{u_{0}}
$$

with $L$ the domain length. The non-dimensional form of the governing equations in differential form are:

$$
\begin{gathered}
\frac{\rho_{c} u_{0}}{L}\left(\frac{\partial \rho}{\partial t}+\nabla \cdot(\rho \boldsymbol{u})\right)=0 \\
\frac{\rho_{c} u_{0}^{2}}{L}\left(\frac{\partial \rho \boldsymbol{u}}{\partial t}+\nabla \cdot(\rho \boldsymbol{u} \otimes \boldsymbol{u}+P \boldsymbol{I}-\boldsymbol{\tau}-\boldsymbol{\xi})\right)=\mathbf{0} \\
\frac{\rho_{c} u_{0}^{3}}{L}\left(\frac{\partial \rho E}{\partial t}+\nabla \cdot\left((\rho E+P) \boldsymbol{u}-(\boldsymbol{\tau}+\boldsymbol{\xi}) \cdot \boldsymbol{u}+\boldsymbol{q}+\boldsymbol{j}_{E}\right)\right)=0
\end{gathered}
$$

It is assumed that the fluid is Newtonian and that the so-called Stokes hypothesis holds. The viscous stress tensor is then expressed as:

$$
\boldsymbol{\tau}=\frac{1}{\rho_{c} u_{0}^{2}} \tilde{\boldsymbol{\tau}}=\frac{1}{R e}\left(\nabla \boldsymbol{u}+\nabla \boldsymbol{u}^{T}-\frac{2}{3} \nabla \cdot \boldsymbol{u I}\right), \quad R e=\frac{\rho_{c} u_{0} L}{\mu}
$$

with $R e$ the Reynolds number and $\mu$ the dynamic viscosity of the fluid. Surface viscosity effects are neglected, which is a common assumption for an interface in a single component fluid without surfactants [39]. The capillary stress tensor is expressed in terms of the local mass density and its spatial derivatives:

$$
\boldsymbol{\xi}=\frac{1}{\rho_{c} u_{0}^{2}} \tilde{\boldsymbol{\xi}}=\frac{1}{W e}\left(\left(\rho \nabla^{2} \rho+\frac{1}{2}|\nabla \rho|^{2}\right) \boldsymbol{I}-\nabla \rho \otimes \nabla \rho\right), \quad W e=\frac{u_{0}^{2} L^{2}}{\rho_{c} K}
$$

with $W e$ the Weber number and $K$ the capillary coefficient. This expression for the capillary stress tensor is a simplified version of the original formulation proposed by Korteweg, which was derived by Papatzacos [27]. The capillary coefficient is directly related to the surface tension coefficient and may depend on the temperature and mass density, but not on the gradient of the mass density [29]. It can be convenient to express scaling relationships in terms of the Ohnesorge number, as it eliminates the effects of inertia and the need for a velocity and length scale. The Ohnesorge number represents the ratio between viscous and surface tension effects:

$$
O h=\frac{\sqrt{W e}}{R e}=\frac{\mu}{\sqrt{\rho_{c}^{3} K}}
$$

The heat flux is expressed as:

$$
\boldsymbol{q}=\frac{1}{\rho_{c} u_{0}^{3}} \tilde{\boldsymbol{q}}=\frac{-8 c_{v}}{3 \operatorname{RePr}} \nabla T, \quad \operatorname{Pr}=\frac{\mu c_{v}}{\lambda}, \quad c_{v}=\frac{1}{R} \tilde{c_{v}}
$$


with $\operatorname{Pr}$ the Prandtl number, $T$ the temperature, $c_{v}$ the non-dimensional specific heat at constant volume, $\lambda$ the coefficient of thermal conductivity of the fluid and $R$ the universal gas constant.

Dunn and Serrin [10] showed that the original formulation of the capillary stress tensor is incompatible with the Clausius-Duhem inequality if the total energy balance equation is not extended with an additional term. This term represents the effect of "interstitial working", which is a form of mechanical work done by the movement of the interface and is expressed as:

$$
\boldsymbol{j}_{E}=\frac{1}{\rho_{c} u_{0}^{3}} \tilde{\boldsymbol{j}}_{E}=\frac{1}{W e}(\rho \nabla \cdot \boldsymbol{u}) \nabla \rho
$$

The energy density is defined as:

$$
\rho E=\rho e+\frac{1}{2} \rho|\boldsymbol{u}|^{2}+\frac{1}{2 W e}|\nabla \rho|^{2}
$$

which represents the sum of the internal energy, kinetic energy and interfacial energy. The expression for the internal energy depends on the equation of state of the fluid.

\subsection{Van der Waals equation of state}

The Van der Waals equation of state [44] represents the relation between the thermodynamic fluid properties or state variables: temperature, pressure and density. It is a cubic equation of state and takes the form:

$$
\tilde{P}=\frac{R \tilde{T} \tilde{\rho}}{1-b \tilde{\rho}}-a \tilde{\rho}^{2}
$$

with $\tilde{P}$ the pressure, $\tilde{T}$ the temperature and $R$ the specific gas constant. The coefficients $a$ and $b$ represent the average attraction between the molecules and the volume excluded by the molecules. The expressions for mass density, pressure and temperature at the critical point can be determined by solving for $\rho$ the following equation:

$$
\frac{\partial \tilde{P}}{\partial \tilde{\rho}}=\frac{\partial^{2} \tilde{P}}{\partial \tilde{\rho}^{2}}=0
$$

which gives the mass density, temperature and pressure at the critical point expressed in the coefficients $a$ and $b$ and the specific gas constant $R$ :

$$
\rho_{c}=\frac{1}{3 b} \quad T_{c}=\frac{8 a}{27 R b} \quad P_{c}=\frac{a}{27 b^{2}}
$$

Although $a, b$ and $R$ differ for every fluid considered, the Van der Waals equation can be recast into a species-independent form with the expressions given above, which yields the non-dimensional Van der Waals equation of state:

$$
P=\frac{8 T \rho}{3-\rho}-3 \rho^{2}
$$

with $T$ the non-dimensional temperature and $\rho$ the non-dimensional mass density. It can be convenient to replace the mass density by the specific volume, $\rho=\frac{1}{v}$, which yields:

$$
P=\frac{8 T}{3 v-1}-\frac{3}{v^{2}}
$$

The internal energy is a function of the local temperature and local mass density. The general equation of state for specific internal energy reads [29]:

$$
e(\rho, T)=\int c_{v} d T+\int\left(P(\rho, T)-\left.T \frac{\partial P}{\partial T}\right|_{\rho}\right) \frac{d \rho}{\rho^{2}}
$$

for a Van der Waals fluid this expression reduces to:

$$
e=\frac{8}{3} \tilde{c}_{v} T-3 \rho
$$

At or above the critical point a fluid can only exist as a one-phase fluid and it is not possible to distinguish between two different phases. Below the critical point the fluid can co-exist as two different phases separated 
by an interface, where the transition region between the phases is called the multiphase region. In the Van der Waals equation the isotherms below the critical point have a wiggle in the multiphase region. By positioning a horizontal line through this wiggle in such a way that the areas A and B (Figure 3) are equal, the vapor-liquid equilibrium can be found [2]. This technique is called the Maxwell equal area rule and it can be mathematically expressed as:

Find $P_{\text {sat }}$ such that:

$$
\int_{v_{1}}^{v_{2}}\left(P(v)-P_{s a t}\right) d v+\int_{v_{2}}^{v_{3}}\left(P(v)-P_{s a t}\right) d v=0
$$

The vapor-liquid equilibrium is found at the saturation pressure, which is the only pressure at which the liquid and vapor can naturally co-exist and it depends solely on the temperature. The procedure of finding the value of $P_{\text {sat }}$ starts with determining the roots of $P(v)-P_{\text {sat }}$ :

$$
\frac{8 T}{3 v-1}-\frac{3}{v^{2}}-P_{s a t}=0
$$

The three roots $\left[v_{1}, v_{2}, v_{3}\right]$ can be determined numerically with a polynomial root-finding algorithm [38]. The liquid/vapor mass density at the saturation pressure are defined as:

$$
\rho_{v, \text { sat }}=\frac{1}{v_{3}} \quad \text { and } \quad \rho_{l, s a t}=\frac{1}{v_{1}}
$$

with $v_{1}$ being the smallest root and $v_{3}$ the largest.

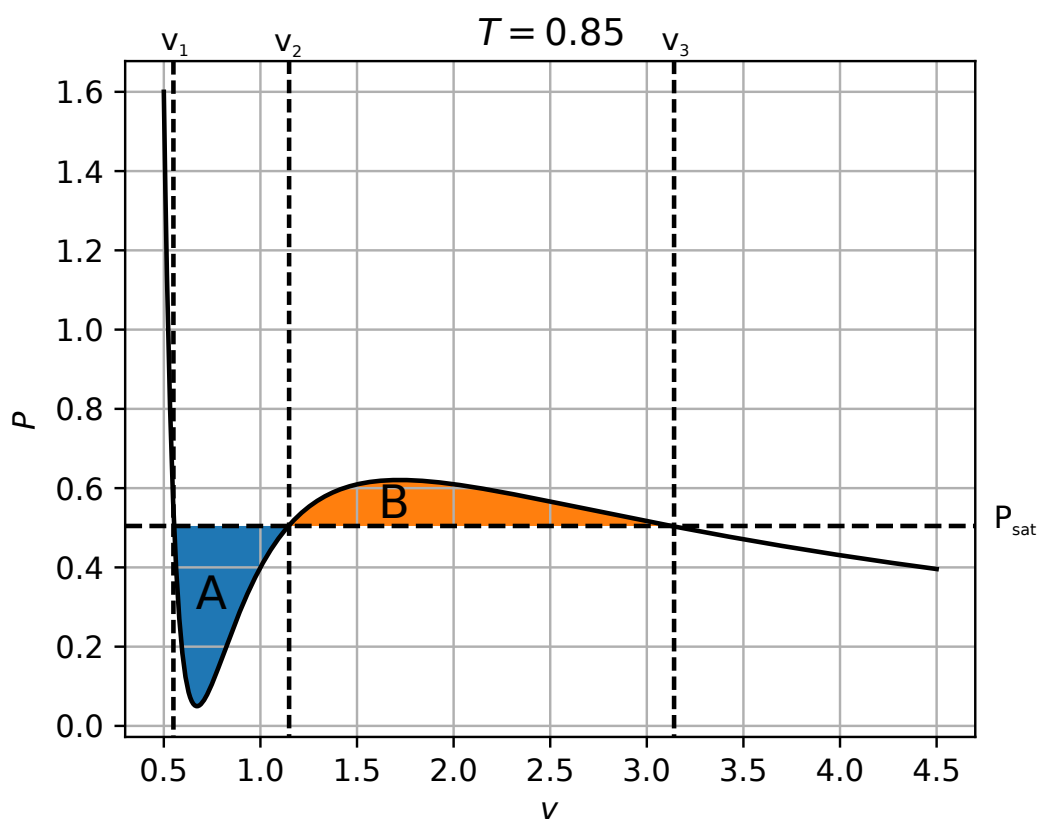

Figure 3: Van der Waals equation of state for $T=0.85$. In satisfying the Maxwell equal area rule we need to find a value for $P_{\text {sat }}$ such that area A equals area B.

A bisection method [37] is used to find the values for $P_{\text {sat }}$ at a range of temperatures. The following expression, together with Table 1, for the non-dimensional coexisting liquid/vapor mass density was obtained by curve fitting the results from this bisection method for $T \in[1.0,0.3]$ and is visualized in Figure 4:

$$
\rho_{l / v, s a t}=1+\sum_{n=1}^{8} a_{n}(1-T)^{n / 2}
$$




\begin{tabular}{|l|l|l|l|l|l|l|l|l|}
\hline$n$ & 1 & 2 & 3 & 4 & 5 & 6 & 7 & 8 \\
\hline$a_{n}$ for $\rho_{l}$ & 2.000 & 0.401 & -0.535 & 0.300 & -0.338 & 0.512 & -0.467 & 0.200 \\
\hline$a_{n}$ for $\rho_{v}$ & -2.000 & 0.370 & 0.877 & -1.945 & 6.883 & -12.474 & 12.261 & -5.032 \\
\hline
\end{tabular}

Table 1: Coefficients belonging to equation 20 obtained by curve fitting the vapor-liquid equilibrium points in the isotherms of the Van der Waals equation

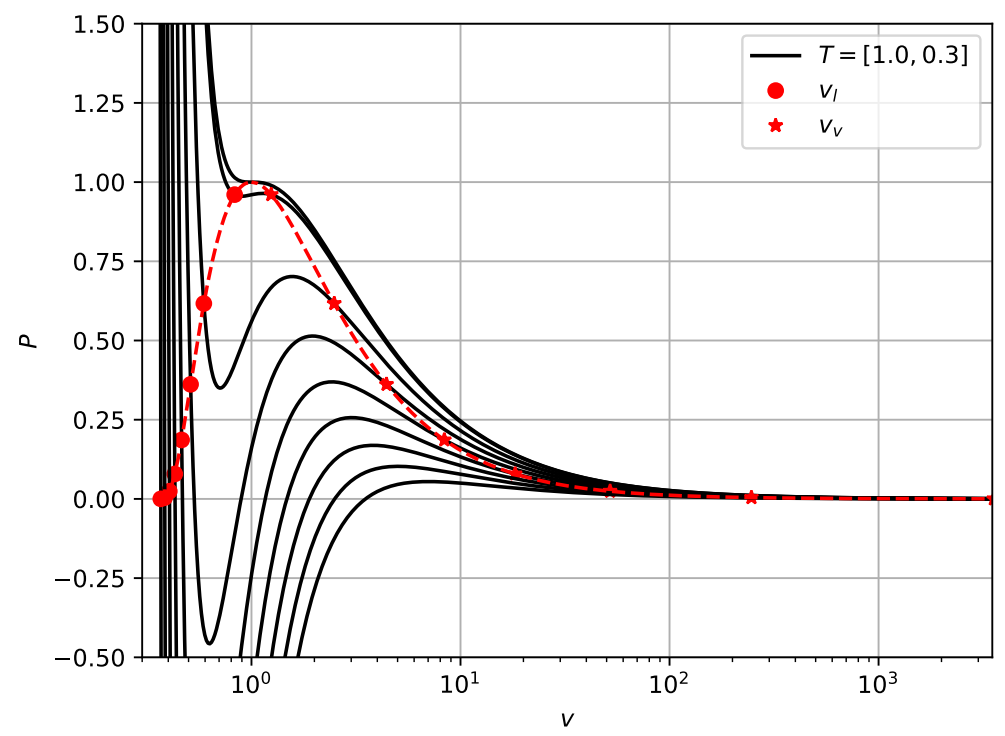

Figure 4: Isotherms of the Van der Waals equation below the critical point. The red dots/stars indicate the coexisting liquid/vapor specific volumes $\left(v=\frac{1}{\rho}\right)$.

\subsection{Helmholtz free energy}

The Helmholtz free energy characterizes thermodynamic properties of a fluid and is determined by the intermolecular forces acting on the molecules that compose the fluid. The intermolecular forces are a competition of both strongly repulsive and weakly attractive forces between the molecules. When the mean field approximation is applied, then the effect of the forces acting on all the individual molecules can be approximated by an averaged thermodynamic potential. Fluid properties such as temperature, pressure and mass density (also called state variables) can be derived from these thermodynamic potentials. The Helmholtz free energy is defined as the useful work obtainable from a closed system at a constant temperature and volume.

In his theory of capillarity Van der Waals proved that the Helmholtz free energy cannot depend only on local variables, such as the local mass density and temperature, since this would cause the thickness of a liquidvapor interface and the surface tension coefficient to be zero. A non-local term needs to be included in the expression for the Helmholtz free energy:

$$
\mathscr{F}=\mathscr{F}_{b}+\frac{1}{2 W e}|\nabla \rho|^{2}, \quad W e=\frac{u_{0}^{2} L^{2}}{\rho_{c} K}
$$

where $\mathscr{F}_{b}$ refers to the bulk of the two phases and $|\nabla \rho|^{2}$ represents the non-local contribution. The expression for the Weber number contains the the capillary coefficient $K$. Its value can be derived from accurate measurements of the surface tension coefficient of a liquid-vapor interface. For a Van der Waals fluid the expression for the Helmholtz free energy in the the bulk of the two phases can be derived from the Van der Waals equation of state and it takes the form:

$$
\mathscr{F}_{b}(\rho, T)=\frac{16}{3} \rho T \operatorname{arctanh}\left(\frac{2}{3} \rho-1\right)-3 \rho^{2}
$$

Note that this expression has only local variables and is only valid in the absence of a liquid-vapor interface.

The properties of a planar liquid-vapor interface can be derived from the excess of Helmholtz free energy in the multiphase region [5], which is the transition region between the two phases, if a thermodynamic equilibrium 
is assumed. The excess of Helmholtz free energy in the multiphase region is defined as follows:

$$
\Delta \mathscr{F}(\rho, T)=\mathscr{F}_{b}(\rho, T)-\mathscr{F}_{l}(\rho, T)
$$

where $\mathscr{F}_{l}$ is called the bi-tangent (Figure 5$)$ and is defined as:

$$
\mathscr{F}_{l}(\rho, T)=\frac{\mathscr{F}\left(\rho_{l, s a t}, T\right)-\mathscr{F}\left(\rho_{v, s a t}, T\right)}{\rho_{l, s a t}-\rho_{v, s a t}}\left(\rho-\rho_{l, s a t}\right)+\mathscr{F}\left(\rho_{l, s a t}, T\right)
$$

An expression for the mass density profile of a liquid-vapor interface can be found by solving the following equation:

$$
\Delta \mathscr{F}(\rho, T)+\frac{1}{2 W e}\left(\frac{d \rho}{d x}\right)^{2}=0
$$

An approximate solution of this equation can only be found if the temperature is close to the critical point [8]. The approximate solution takes the form of a hyperbolic tangent function:

$$
\rho(x)=\frac{\rho_{l, s a t}+\rho_{v, s a t}}{2}+\frac{\rho_{l, s a t}-\rho_{v, s a t}}{2} \tanh \left(\frac{4\left(x-x_{i}\right)}{L_{i}}\right)
$$

with $x_{i}$ the midpoint location of the interface and $L_{i}$ the interface thickness (Figure 5).

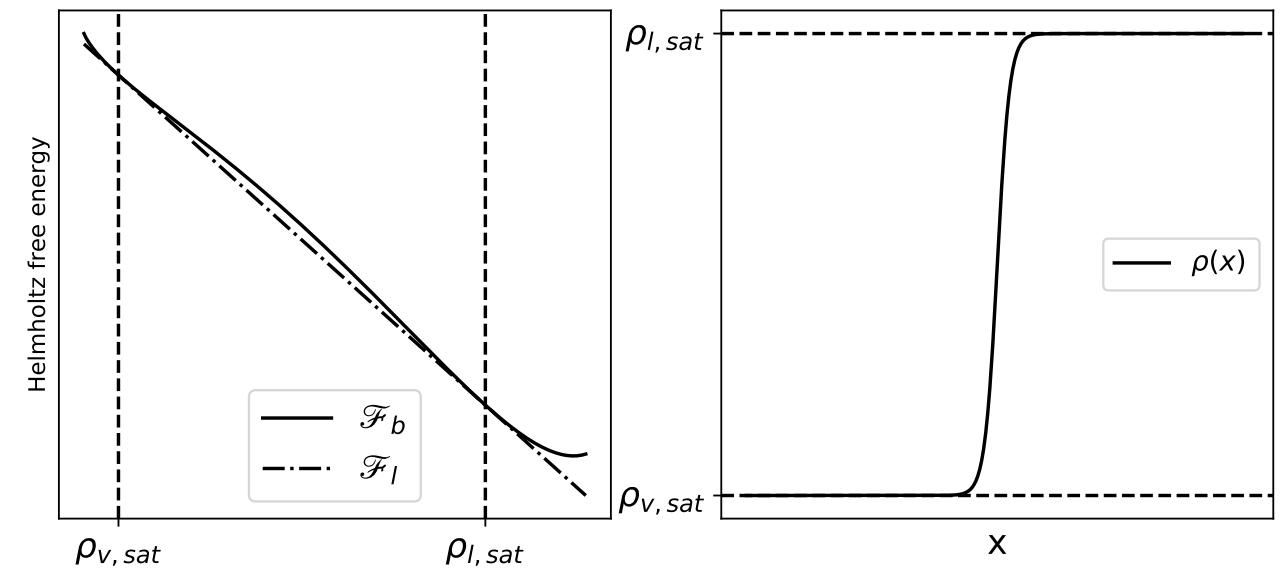

Figure 5: Helmholtz free energy $\mathscr{F}_{b}$, the bi-tangent line $\mathscr{F}_{l}$ and the mass density profile $\rho(x)$ of a liquid-vapor interface for a fixed temperature

Both the surface tension coefficient $\sigma$ and the thickness of the liquid-vapor interface $L_{i}$ can also be determined from the excess free energy function [5]. These expressions also hold further away from the critical point:

$$
\begin{aligned}
\sigma & =\sqrt{\frac{2}{W e}} \int_{\rho_{v, s a t}}^{\rho_{l, s a t}} \sqrt{\Delta \mathscr{F}} d \rho \\
L_{i} & =\sqrt{\frac{2}{W e}} \frac{\left(\rho_{l, s a t}-\rho_{v, s a t}\right)^{2}}{\int_{\rho_{v, s a t}}^{\rho_{l, s a t}} \sqrt{2 \Delta \mathscr{F}} d \rho}
\end{aligned}
$$

With these expressions it becomes possible to determine the proportionality of the surface tension coefficient and interface thickness to the temperature in relation to the critical temperature. For a Van der Waals fluid this proportionality is as follows:

$$
\begin{gathered}
\sigma \propto\left(1-T / T_{c}\right)^{1.5} \\
L_{i} \propto\left(1-T / T_{c}\right)^{-0.5}
\end{gathered}
$$

It is well known that although a Van der Waals fluid behaves qualitatively similar to real fluids, exact numerical comparison with experimental data show it to be an inaccurate fluid model. For example, for most real fluids the proportionality shown above is closer to [6]:

$$
\begin{gathered}
\sigma \propto\left(1-T / T_{c}\right)^{1.2} \\
L_{i} \propto\left(1-T / T_{c}\right)^{-0.6}
\end{gathered}
$$


The first major improvement to the Van der Waals equation was introduced by Redlich and Kwong [32], where the attractive term of the equation becomes temperature dependent. This modification improves the accuracy of the equation primarily above the critical point, below the critical point and especially in the liquid phase region the accuracy is still poor. Further modifications and extensions were introduced by numerous authors, eventually culminating in the PRSV2 equation. The PRSV2 equation is the second version of the Peng-Robinson-Stryjek-Vera equation [40] and is generally considered to be the most advanced cubic equation of state. It is an extended version of the Peng-Robinson equation of state which can be used to accurately calculate vapor-liquid equilibria. However, its direct use in DIM is unpractical, since the expression for the internal energy is prohibitively complex for use in numerical simulations if the PRSV2 equation is applied. Additionally, is was also noted that although the PRSV2 equation is more accurate for calculating vaporliquid equilibria, comparison with experimental data shows that the simpler Redlich-Kwong equation is more accurate when calculating the surface tension coefficient [45].

Direct modification of the Helmholtz free energy function can extend the temperature range in which the DIM described in the present study can be used. This range is severely limited by the fact that the liquidvapor interface becomes very thin when the temperature is far from the critical point. This leads to a severe constraint on the grid spacing, since a sufficient number of grid points is needed to resolve the interface region (at least 10 grid points across the interface thickness). If this constraint is combined with the use of a fixed grid, then the number of grid points needed in the computational domain quickly becomes excessively high when the temperature is far from the critical point. Jamet et al. [16] demonstrated a technique for artificial enlargement of the liquid-vapor interface thickness in a thermodynamically consistent manner, by using the double well-potential function to approximate the excess of Helmholtz free energy in the multiphase region. This technique eliminates the constraint on the grid spacing, but it is currently only applicable to a system under isothermal conditions and therefore it was not used in the present study.

\subsection{Laplace Pressure}

The Laplace pressure is the pressure difference between the inside and outside of either a droplet or a bubble. The pressure difference is caused by the surface tension and can be calculated with the Young-Laplace equation, which for a spherical droplet or bubble with radius $\mathrm{R}$ reads in three spatial dimensions:

$$
\left|P_{l}-P_{v}\right|=\frac{2 \sigma_{l v}}{R}
$$

with $\sigma_{l v}$ the liquid-vapor surface tension coefficient. The Young-Laplace equation represents a simplified form of the normal momentum jump condition which is permitted when velocity gradients near the interface are negligible, as compared to pressure changes [39]. The liquid and vapor pressures can be calculated with the following expressions [6]:

$$
P_{v}=P_{s a t}+\eta \frac{\rho_{v, s a t}}{\rho_{l, s a t}-\rho_{v, s a t}} \frac{2 \sigma_{l v}}{R}
$$

and

$$
P_{l}=P_{s a t}+\eta \frac{\rho_{l, s a t}}{\rho_{l, s a t}-\rho_{v, s a t}} \frac{2 \sigma_{l v}}{R}
$$

with $P_{\text {sat }}=P_{\text {sat }}(T)$ and $\eta$ :

$$
\eta= \begin{cases}+1 & \text { for a droplet } \\ -1 & \text { for a bubble }\end{cases}
$$

The effect of the Laplace pressure on the liquid and vapor mass density inside and outside of a droplet or a bubble is determined with an isotherm of the Van der Waals equation, as visualized in Figure 6. A polynomial root-finding algorithm is used to determine the liquid and vapor mass density at the corresponding pressures. 

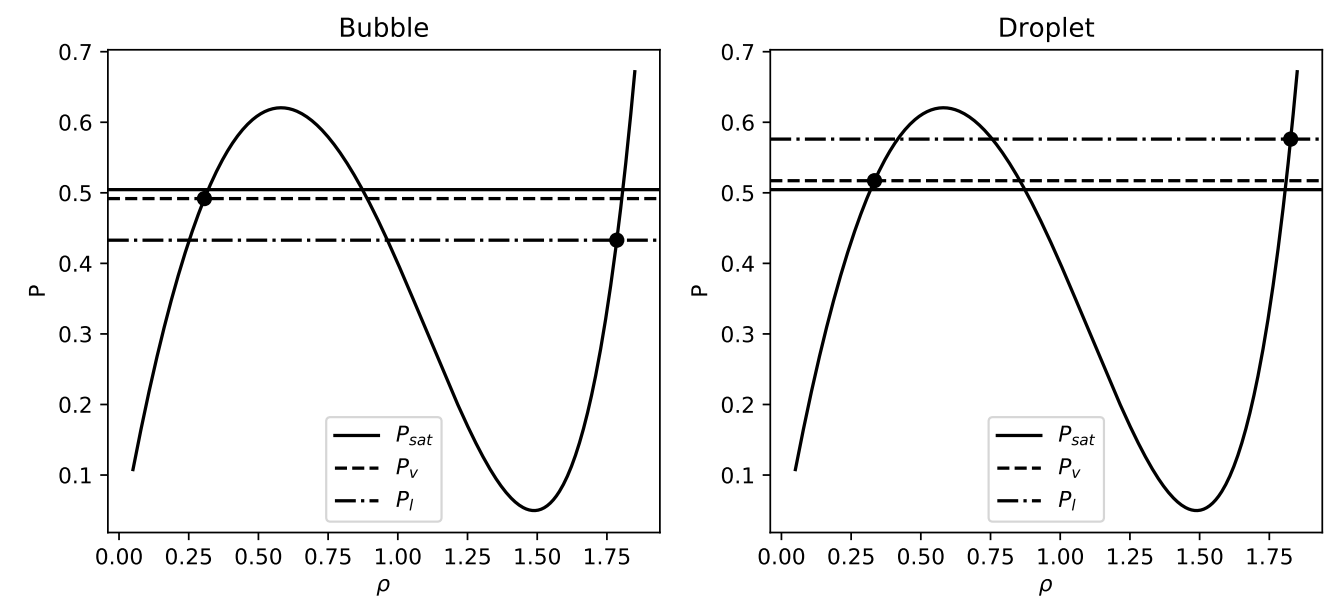

Figure 6: The effect of the Laplace pressure on the liquid and vapor mass density inside and outside of either a droplet or a bubble is determined with an isotherm of the Van der Waals equation [6]. The pressure difference is calculated with the Young-Laplace equation and a polynomial root-finding algorithm is used to determine the liquid and vapor mass density.

\section{Numerical method}

The discretization method used in the present study is the same as the one used by Pecenko et al. [28, 29] and Desmarais and Kuerten [9], which is based on a discretization technique developed by Cockburn and Gau [7]. It uses a second-order accurate finite-volume method for spatial discretization and a third-order accurate TVD Runge-Kutta time integration method developed by Shu and Osher [36]. Centered difference schemes are employed to calculate the derivatives in the flux expressions. The finite volume method is preferred over other discretization methods since it is strictly conservative.

Numerical methods for solving the governing equations of DIM have to cope with additional difficulties when compared to single-phase flow simulations. One such difficulty is that the capillary stress tensor causes dispersive behavior of the solution, since it contains a second-order spatial derivative of the mass density. Also, the Van der Waals equation of state has a non-convex part which causes a mixed hyperbolic-elliptic nature of the governing equations. This prevents the use of upwind based discretization methods, which are commonly used for compressible flow simulations.

\subsection{NSK equations in Cartesian coordinates}

The governing equations are written as a set of conservation equations in three dimensions:

$$
\frac{\partial \boldsymbol{U}}{\partial t}+\frac{\partial \boldsymbol{F}}{\partial x}+\frac{\partial \boldsymbol{G}}{\partial y}+\frac{\partial \boldsymbol{H}}{\partial z}=\mathbf{0}
$$

with $\boldsymbol{U}$ the vector containing the conserved variables and $\boldsymbol{F}, \boldsymbol{G}$, and $\boldsymbol{H}$ the flux vectors in $\mathrm{x}, \mathrm{y}$ and $\mathrm{z}$ direction, expressed as:

$$
\begin{gathered}
\boldsymbol{U}=\left(\begin{array}{c}
\rho \\
\rho u \\
\rho v \\
\rho w \\
\rho E
\end{array}\right) \quad \boldsymbol{F}=\left(\begin{array}{c}
\rho u \\
\rho u^{2}+P-\tau_{x x}-\xi_{x x} \\
\rho u v-\tau_{x y}-\xi_{x y} \\
\rho u w-\tau_{x z}-\xi_{x z} \\
(\rho E+P) u-\left(\tau_{x x}+\xi_{x x}\right) u-\left(\tau_{x y}+\xi_{x y}\right) v-\left(\tau_{x z}+\xi_{x z}\right) w+q_{x}+j_{E x}
\end{array}\right) \\
\boldsymbol{G}=\left(\begin{array}{c}
\rho v \\
\rho v u-\tau_{y x}-\xi_{y x} \\
\rho v^{2}+P-\tau_{y y}-\xi_{y y} \\
\rho v w-\tau_{y z}-\xi_{y z} \\
(\rho E+P) v-\left(\tau_{y x}+\xi_{y x}\right) u-\left(\tau_{y y}+\xi_{y y}\right) v-\left(\tau_{y z}+\xi_{y z}\right) w+q_{y}+j_{E y}
\end{array}\right)
\end{gathered}
$$




$$
\boldsymbol{H}=\left(\begin{array}{c}
\rho w \\
\rho w u-\tau_{z x}-\xi_{z x} \\
\rho w v-\tau_{z y}-\xi_{z y} \\
\rho w^{2}+P-\tau_{z z}-\xi_{z z} \\
(\rho E+P) w-\left(\tau_{z x}+\xi_{z x}\right) u-\left(\tau_{z y}+\xi_{z y}\right) v-\left(\tau_{z}+\xi_{z z}\right) w+q_{z}+j_{E z}
\end{array}\right)
$$

with the viscous stress tensor:

$$
\begin{gathered}
\tau_{x x}=\frac{1}{R e}\left[\frac{4}{3} \frac{\partial u}{\partial x}-\frac{2}{3} \frac{\partial v}{\partial y}-\frac{2}{3} \frac{\partial w}{\partial z}\right] \\
\tau_{y y}=\frac{1}{R e}\left[\frac{4}{3} \frac{\partial v}{\partial y}-\frac{2}{3} \frac{\partial u}{\partial x}-\frac{2}{3} \frac{\partial w}{\partial z}\right] \\
\tau_{z z}=\frac{1}{R e}\left[\frac{4}{3} \frac{\partial w}{\partial z}-\frac{2}{3} \frac{\partial u}{\partial x}-\frac{2}{3} \frac{\partial v}{\partial y}\right] \\
\tau_{x y}=\tau_{y x}=\frac{1}{R e}\left[\frac{\partial u}{\partial y}+\frac{\partial v}{\partial x}\right] \\
\tau_{x z}=\tau_{z x}=\frac{1}{R e}\left[\frac{\partial w}{\partial x}+\frac{\partial u}{\partial z}\right] \\
\tau_{y z}=\tau_{z y}=\frac{1}{R e}\left[\frac{\partial w}{\partial y}+\frac{\partial v}{\partial z}\right]
\end{gathered}
$$

the capillary stress tensor:

$$
\begin{gathered}
\xi_{x x}=\frac{1}{W e}\left[\rho\left(\frac{\partial^{2} \rho}{\partial x^{2}}+\frac{\partial^{2} \rho}{\partial y^{2}}+\frac{\partial^{2} \rho}{\partial z^{2}}\right)+\frac{1}{2}\left(-\left(\frac{\partial \rho}{\partial x}\right)^{2}+\left(\frac{\partial \rho}{\partial y}\right)^{2}+\left(\frac{\partial \rho}{\partial z}\right)^{2}\right)\right] \\
\xi_{y y}=\frac{1}{W e}\left[\rho\left(\frac{\partial^{2} \rho}{\partial x^{2}}+\frac{\partial^{2} \rho}{\partial y^{2}}+\frac{\partial^{2} \rho}{\partial z^{2}}\right)+\frac{1}{2}\left(\left(\frac{\partial \rho}{\partial x}\right)^{2}-\left(\frac{\partial \rho}{\partial y}\right)^{2}+\left(\frac{\partial \rho}{\partial z}\right)^{2}\right)\right] \\
\xi_{z z}=\frac{1}{W e}\left[\rho\left(\frac{\partial^{2} \rho}{\partial x^{2}}+\frac{\partial^{2} \rho}{\partial y^{2}}+\frac{\partial^{2} \rho}{\partial z^{2}}\right)+\frac{1}{2}\left(\left(\frac{\partial \rho}{\partial x}\right)^{2}+\left(\frac{\partial \rho}{\partial y}\right)^{2}-\left(\frac{\partial \rho}{\partial z}\right)^{2}\right)\right] \\
\xi_{x y}=\xi_{y x}=-\frac{1}{W e} \frac{\partial \rho}{\partial x} \frac{\partial \rho}{\partial y} \\
\xi_{x z}=\xi_{z x}=-\frac{1}{W e} \frac{\partial \rho}{\partial x} \frac{\partial \rho}{\partial z} \\
\xi_{y z}=\xi_{z y}=-\frac{1}{W e} \frac{\partial \rho}{\partial y} \frac{\partial \rho}{\partial z}
\end{gathered}
$$

the heat flux:

$$
\begin{aligned}
& q_{x}=\frac{-8 c_{v}}{3 \operatorname{RePr}} \frac{\partial T}{\partial x} \\
& q_{y}=\frac{-8 c_{v}}{3 \operatorname{RePr} r} \frac{\partial T}{\partial y} \\
& q_{z}=\frac{-8 c_{v}}{3 \operatorname{RePr} r} \frac{\partial T}{\partial z}
\end{aligned}
$$

and the interstitial working flux:

$$
\begin{aligned}
& j_{E x}=\frac{1}{W e} \rho\left(\frac{\partial u}{\partial x}+\frac{\partial v}{\partial y}+\frac{\partial w}{\partial z}\right) \frac{\partial \rho}{\partial x} \\
& j_{E y}=\frac{1}{W e} \rho\left(\frac{\partial u}{\partial x}+\frac{\partial v}{\partial y}+\frac{\partial w}{\partial z}\right) \frac{\partial \rho}{\partial y} \\
& j_{E z}=\frac{1}{W e} \rho\left(\frac{\partial u}{\partial x}+\frac{\partial v}{\partial y}+\frac{\partial w}{\partial z}\right) \frac{\partial \rho}{\partial z}
\end{aligned}
$$

\subsection{Spatial and temporal discretization}

The variables and their derivatives at the edges of the cells are calculated using centered difference schemes. The schemes used for the edges in $\mathrm{x}$-direction, with $u$ an arbitrary variable:

$$
u_{i+\frac{1}{2}, j, k}=\frac{u_{i+1, j, k}+u_{i, j, k}}{2}
$$




$$
\begin{gathered}
\left.\frac{\partial u}{\partial x}\right|_{i+\frac{1}{2}, j, k}=\frac{u_{i+1, j, k}-u_{i, j, k}}{\Delta x} \\
\left.\frac{\partial u}{\partial y}\right|_{i+\frac{1}{2}, j, k}=\frac{u_{i+\frac{1}{2}, j+1, k}-u_{i+\frac{1}{2}, j-1, k}}{2 \Delta y} \\
\left.\frac{\partial u}{\partial z}\right|_{i+\frac{1}{2}, j, k}=\frac{u_{i+\frac{1}{2}, j, k+1}-u_{i+\frac{1}{2}, j, k-1}}{2 \Delta z} \\
\left.\frac{\partial^{2} u}{\partial x^{2}}\right|_{i+\frac{1}{2}, j, k}=\frac{u_{i+2, j, k}-u_{i+1, j, k}-u_{i, j, k}+u_{i-1, j, k}}{2 \Delta x^{2}} \\
\left.\frac{\partial^{2} u}{\partial y^{2}}\right|_{i+\frac{1}{2}, j, k}=\frac{u_{i+\frac{1}{2}, j+1, k}-2 u_{i+\frac{1}{2}, j, k}-u_{i+\frac{1}{2}, j-1, k}}{\Delta y^{2}} \\
\left.\frac{\partial^{2} u}{\partial z^{2}}\right|_{i+\frac{1}{2}, j, k}=\frac{u_{i+\frac{1}{2}, j, k+1}-2 u_{i+\frac{1}{2}, j, k}-u_{i+\frac{1}{2}, j, k-1}}{\Delta z^{2}}
\end{gathered}
$$

A set of equivalent schemes are used for the edges in $y$ and $z$ direction. The differential equations are discretized on a uniform Cartesian grid. The semi-discrete system of equations becomes:

$$
\frac{d}{d t} \boldsymbol{U}_{i, j, k}=-\frac{1}{\Delta x}\left(\boldsymbol{F}_{i+\frac{1}{2}, j, k}-\boldsymbol{F}_{i-\frac{1}{2}, j, k}\right)-\frac{1}{\Delta y}\left(\boldsymbol{G}_{i, j+\frac{1}{2}, k}-\boldsymbol{G}_{i, j-\frac{1}{2}, k}\right)-\frac{1}{\Delta z}\left(\boldsymbol{H}_{i, j, k+\frac{1}{2}}-\boldsymbol{H}_{i, j, k-\frac{1}{2}}\right)
$$

Numerical instabilities due to the non-monotonic behavior caused by the region of negative compressibility in the Van der Waals isotherms are prevented by using a third-order accurate TVD Runge-Kutta time-integration scheme [36]:

$$
\begin{gathered}
U_{i, j, k}^{(1)}=U_{i, j, k}^{(n)}+\Delta t \frac{d}{d t} \boldsymbol{U}_{i, j, k}^{(n)} \\
U_{i, j, k}^{(2)}=\frac{3}{4} U_{i, j, k}^{(n)}+\frac{1}{4}\left[U_{i, j, k}^{(1)}+\Delta t \frac{d}{d t} U_{i, j, k}^{(1)}\right] \\
U_{i, j, k}^{(n+1)}=\frac{1}{3} U_{i, j, k}^{(n)}+\frac{2}{3}\left[U_{i, j, k}^{(2)}+\Delta t \frac{d}{d t} \boldsymbol{U}_{i, j, k}^{(2)}\right]
\end{gathered}
$$

The time step $\Delta t$ has to satisfy a number of conditions to ensure stability. For the $x$ direction these conditions are [9]:

$$
\begin{gathered}
\left(\frac{|u|+u_{0}}{2 \Delta x}+\sqrt{\frac{\rho}{4 W e \Delta x^{4}}}\right) \Delta t \leq 1.5 \\
\frac{4 \Delta t}{\rho \operatorname{RePr} \Delta x^{2}} \leq 2.5 \\
\frac{8 \Delta t}{3 \rho \operatorname{Re} \Delta x^{2}} \leq 2.5
\end{gathered}
$$

where $\rho$ and $u$ are the local mass density and velocity. For the $y$ and $z$ direction the conditions are similar.

\subsection{Initialization and boundary conditions}

The computational domain $\Omega$ is constructed as a cube with length $L=1.0$ in each spatial dimension:

$$
\Omega=(0.0,1.0)^{3} \subset \mathbb{R}^{3}
$$

The computational grid is Cartesian and uniform with 200 grid points in each direction, resulting in a total of 8 million grid points. Simulations on finer grids showed no significant differences in the simulations results. Symmetry boundary conditions are imposed on all the domain boundaries.

The initial mass density field is constructed with a hyperbolic tangent function in the shape of two identical spherical droplets (Figure 8) with a diameter of $D=0.2$, positioned left and right of the center of the domain:

$$
\rho(x, y, z)_{t=0}=\rho_{v}+\frac{1}{2}\left|\rho_{l}-\rho_{v}\right|\left(\tanh \left(\frac{4\left(d_{1}(x, y, z)-r_{d}\right)}{L_{i}}\right)+\tanh \left(\frac{4\left(d_{2}(x, y, z)-r_{d}\right)}{L_{i}}\right)\right)
$$


with $\rho_{v}, \rho_{l}$ the vapor and liquid mass density, $r_{d}=\frac{D}{2}$ the droplet radius, $L_{i}$ the interface thickness and $d_{1}, d_{2}$ :

$$
d_{1}(x, y, z)=\left\|\left(\begin{array}{c}
0.5-2 r_{d} \\
0.5 \\
0.5+X / 2
\end{array}\right)-\left(\begin{array}{l}
x \\
y \\
z
\end{array}\right)\right\| \quad d_{2}(x, y, z)=\left\|\left(\begin{array}{c}
0.5+2 r_{d} \\
0.5 \\
0.5+X / 2
\end{array}\right)-\left(\begin{array}{l}
x \\
y \\
z
\end{array}\right)\right\|
$$

with $X$ the offset in z-direction between the velocity vectors of the droplets. The velocity vectors are equal in magnitude but opposite in direction (Figure 7)

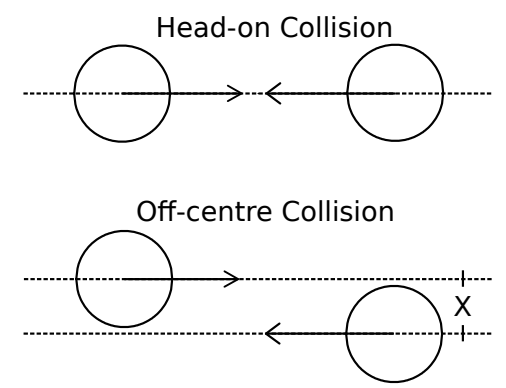

Figure 7: Head-on and off-centre binary droplet collision with $X$ the offset in z-direction between the velocity vectors of the droplets.

The effect of the Laplace pressure on the vapor and liquid mass density of a droplet and its surrounding vapor is visualized in Figure 6. This effect needs to be accounted for in the initialization of the mass density field.

The initial velocity field is also constructed with a hyperbolic tangent function (Figure 8):

$$
\boldsymbol{u}(x, y, z)_{t=0}=\left(\begin{array}{cc}
\frac{v_{d}}{2}\left(1-\tanh \left(4 \frac{d_{1}-r_{d}}{L_{i}}\right)\right) & x<0.5 \\
-\frac{v_{d}}{2}\left(1-\tanh \left(4 \frac{d_{2}-r_{d}}{L_{i}}\right)\right) & x>0.5 \\
0 &
\end{array}\right)
$$

The initial temperature field is uniform and close to the critical point:

$$
T(x, y, z)_{t=0}=0.85 T_{c}
$$

At this temperature the liquid-vapor interface has a thickness of approximately $L_{i}=0.075$, leading to approximately 15 grid points across the interface thickness, which is sufficient for resolving the interface region with minimal discretization error.

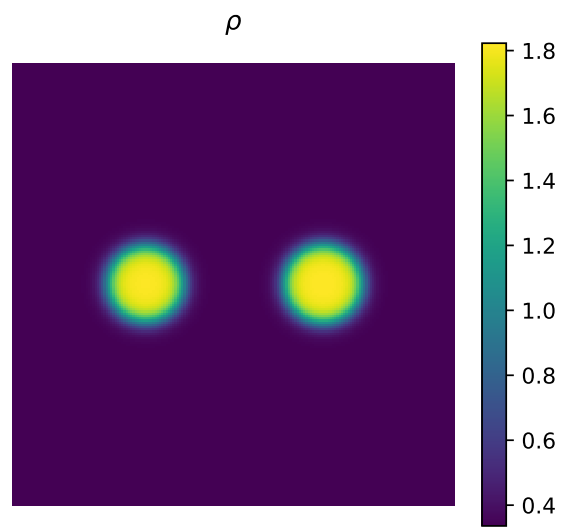

$\vec{u}$

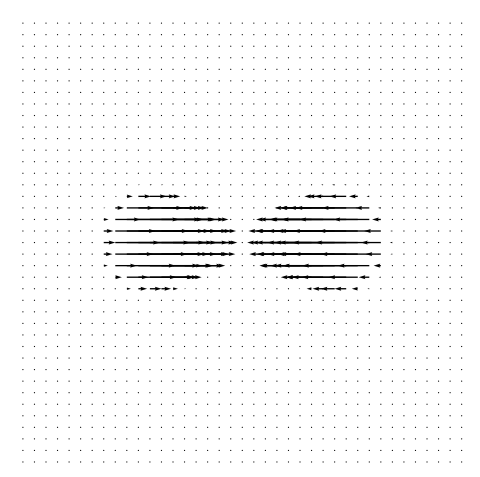

Figure 8: An $x y$ cross-section of the domain at $z=0.5$ showing the initial mass density and velocity field. The domain length equals $L=1.0$ in each spatial dimension, with a total of $200^{3}$ grid points. The droplets have a diameter of $D=0.2$ and the interface has a thickness of approximately $L_{i}=0.075$. Only $1 / 5$ of the total number of grid points were used to produce the vector plot on the right. 


\section{Simulation results}

In this section results are presented obtained from three-dimensional, non-isothermal simulations of droplet collisions. Droplet coalescence and subsequent separation regimes are observed for both head-on and off-centre droplet collisions at various impact velocities.

In the visualizations of the simulation results the liquid-vapor interface is represented by a two dimensional surface mesh that was constructed using the Lewiner marching cubes algorithm [20], where the mass density field was used as the volume data and the isosurface value was set to the average of the liquid and vapor mass densities at saturation pressure. An overview of the values of the simulation parameters for all the simulation results visualized in this section can be found in Table 2.

\begin{tabular}{|c|c|c|c|c|c|c|c|c|}
\hline Simulation & $R e$ & $W e$ & $\operatorname{Pr}$ & $c_{v}$ & Oh & $v_{r e l}$ & $\mathscr{X}$ & $W e_{c}$ \\
\hline Figure 9 & 300.0 & 6000.0 & 20.0 & 5.0 & 0.258 & 1.5 & 0.0 & 75 \\
Figure 10 & 1250.0 & 6000.0 & 20.0 & 5.0 & 0.062 & 2.2 & 0.0 & 140 \\
Figure 11 & 1000.0 & 6000.0 & 20.0 & 5.0 & 0.078 & 4.0 & 0.0 & 520 \\
Figure 14 & 300.0 & 6000.0 & 20.0 & 5.0 & 0.258 & 3.0 & 0.5 & 130 \\
\hline
\end{tabular}

Table 2: Overview of the values of the simulation parameters for all the simulation results visualized in this section.

\subsection{Head-on collisions}

When the droplets touch, a small liquid bridge is formed which is followed by its subsequent broadening. Coalescence occurs and the coalesced droplets spread outward in the radial direction, which first forms a flat disk possibly followed by a radially expanding ring. This expansion is counteracted by the surface tension forces and damped by the viscous forces. After reaching the maximum radial spread, the ring/disk starts to contract radially. This is followed by an elongation in the axial direction, which generates a round-ended cylindrical droplet (Figure 9 and 10). The viscous dissipation damps the reflexive movement of the droplet. If the damping is sufficiently strong, then the coalesced droplets only oscillate with diminishing amplitude until a perfectly spherical droplet remains, as can be seen in Figure 9. If viscous damping is insufficient, then a reflexive separation can occur. The round-ended cylindrical droplet, which is formed due to the reflexive movement, elongates to such an extent that it ruptures into two separate droplets of equal size, as can be seen in figure 10 .

the.

Figure 9: Example of a perfect head-on collision resulting in permanent coalescence. $O h=0.258, W e_{c}=$ $130, \mathscr{X}=0.0$ 


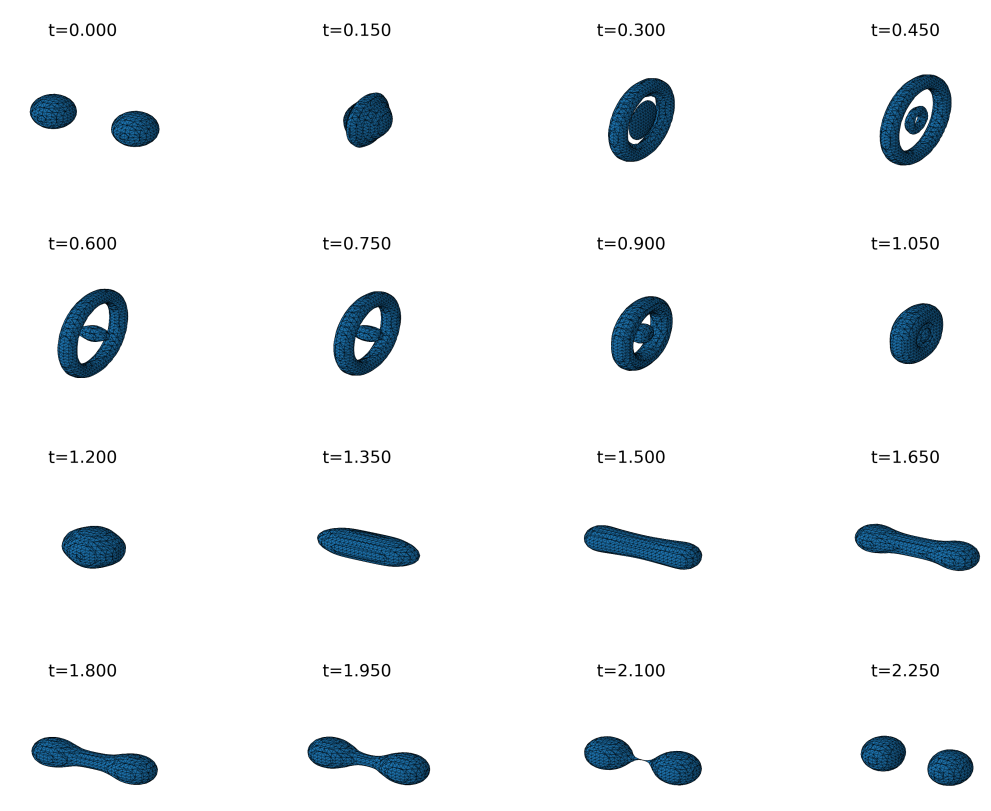

Figure 10: Example of a perfect head-on collision resulting in a reflexive separation. $O h=0.062, W e_{c}=$ $140, \mathscr{X}=0.0$

Figure 11 shows a collision with a much higher relative velocity. The radial spreading of the coalesced droplets is so large that when this ring starts to contract, it is no longer able to contract to a single droplet. The liquid ring starts to break-up soon after it starts to contract and a ring-shaped cluster of 8 smaller droplets is formed. The breakup process can be attributed to the Plateau-Rayleigh (PR) instability, a phenomenon where small-amplitude disturbances on an interface grow and initiate a breakup of a liquid thread, or in this case a thin liquid ring. The collision causes small-amplitude waves in the mass density and velocity field that propagate towards the boundaries of the domain, where they are reflected because of the symmetry boundary conditions that are imposed. The effect of this phenomenon is negligibly small for most simulations, since it are small-amplitude waves with a diminishing amplitude. However, because of the small width of the ring in this particular simulation, the effect described above is sufficiently large to trigger the PR-instability. Pairam and Fernández-Nieves [26] performed an experimental study on the stability of a thin liquid ring, also called a toroidal droplet. Critical to the stability is the ratio between the mean radius $R$ and the half-width of the ring $a$ (Figure 12) which determines whether the toroidal droplet contracts to a single droplet or it breaks into multiple droplets. In Figure 11 this ratio reaches a maximum of approximately 14.5, which is in accordance with the findings of Pairam and Fernández-Nieves who observed a breakup of a toroidal droplet into 8 smaller droplets for $\frac{R}{a}$ of approximately 14 .

The regime of droplet bouncing was not observed in any of the simulations. Whether or not droplets bounce is determined by the properties of the ambient gas. As the two droplets approach each other, a gas layer is trapped between them. This compressed gas layer can prevent the contact of the droplet surfaces, causing the droplets to deform but not to touch. The reflexive properties of the interface then cause the droplets to bounce apart. In experimental investigations droplet bouncing is normally observed when the droplets are surrounded by a gas of a different component at a sufficiently high pressure [30]. In the present study there is an important difference: the liquid droplets are surrounded by vapor comprised of the same molecules as the liquid, since the NSK equations can only be used for a single component fluid. Therefore, the vapor trapped between the droplets is able to condense and can be absorbed by the droplets. It is therefore less likely that droplet bouncing will be observed when the NSK equations are used.

The initial temperature field is uniform. Variations in the temperature field are caused by the movement of the liquid-vapor interface due to viscous dissipation of kinetic energy and the effect of interstitial working. However, for a moving droplet the most dominant temperature variations are caused by condensation and evaporation. In front of the droplet the temperature is increased due to condensation and at the back the temperature is decreased due to evaporation. In between the droplets compression of the vapor causes a strong increase in local temperature, as can be observed in figure 13. Coalescence of the droplets is facilitated by the increase in local temperature, since the surface tension is lower at higher temperatures. At or above 

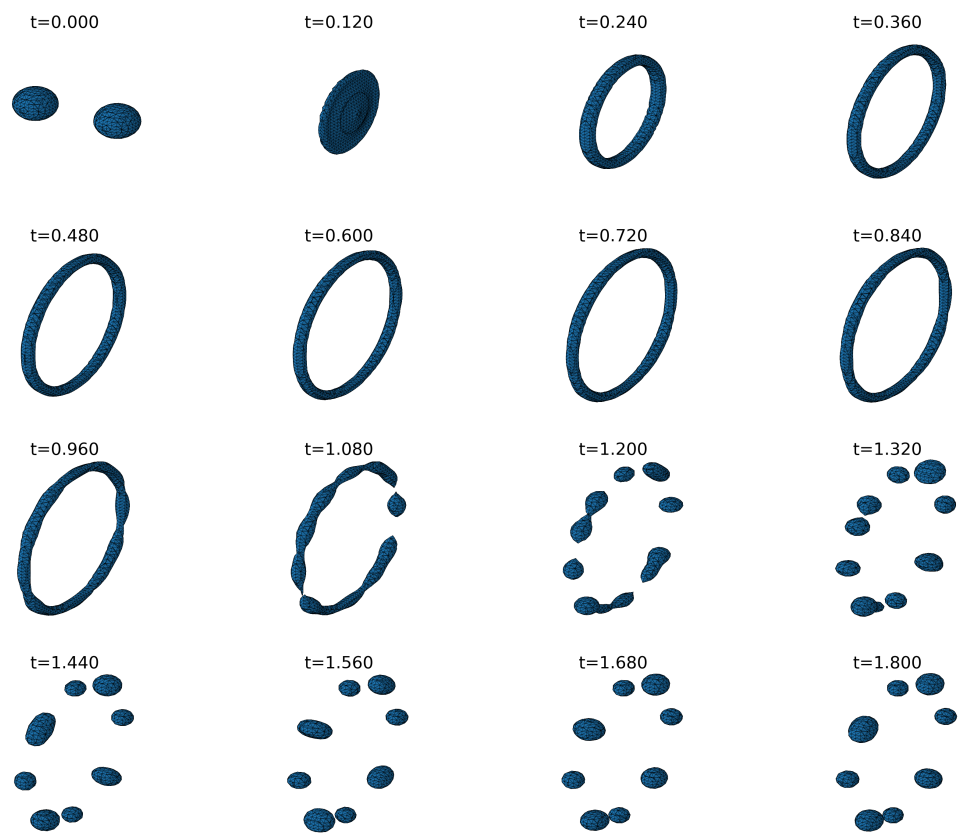

Figure 11: Example of a perfect head-on collision resulting in a toroidal droplet breakup. The colliding droplets to disintegrate into a cluster of many smaller droplets. $O h=0.078, W e_{c}=520, \mathscr{X}=0.0$

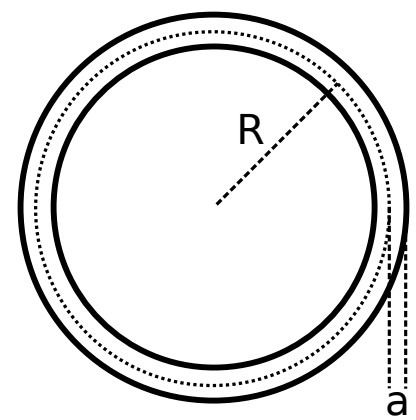

Figure 12: Schematic sketch of a thin liquid ring, also called a toroidal droplet [26], with $R$ mean the radius and $a$ the half-width of the ring.

the critical point $(T>=1.0)$ the surface tension is zero and the liquid-vapor interface no longer exist, at which point coalescence happens automatically.

The rate of diffusion of heat is inversely proportional to the value of the Prandtl number (equation 8). Therefore, for high Prandtl numbers temperature variations will be higher because diffusion of heat is reduced. Simulations at lower Prandtl numbers (e.g. $\mathrm{Pr}=0.1$ ) show a reduction in the maximum increase in local temperature in between the droplets. However, even when the local temperature remains below the critical point, coalescence of the colliding droplets is still observed. 

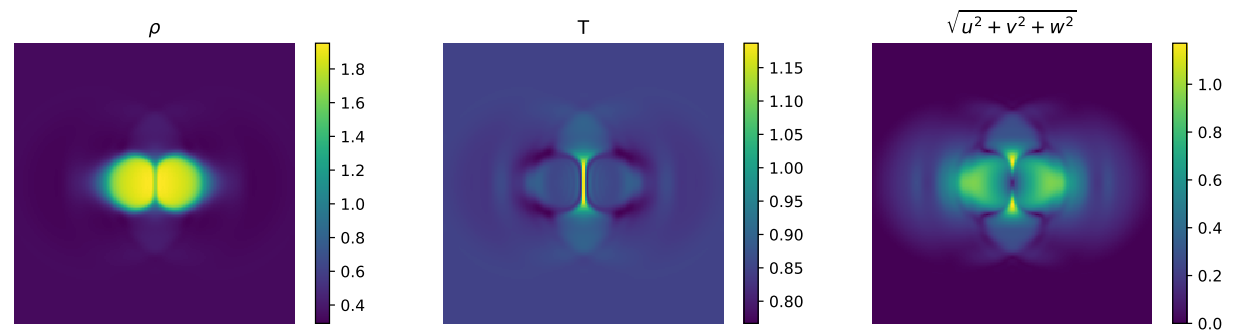

Figure 13: An $x y$ cross-section at $z=0.5$ showing the mass density, temperature and absolute velocity field at $t=0.08$ for the simulation in Figure 10 .

\subsection{Off-centre collisions}

In this section results are presented obtained from a simulation of an off-centre droplet collision with an impact parameter of $\mathscr{X}=0.5$. When two droplets collide with positive/nonzero impact parameters, only a portion of the fluid that comprises the droplets are on colliding trajectories. Although the fluid spreads outwards in the radial direction during the first stage of the collision (in a manner similar to the head-on collision), the ring that is formed is skewed and the fluid is not evenly distributed over the ring. The inertia of the fluid causes the ring to be stretched in the directions of the initial trajectories of the droplets. The stretching is counteracted by the surface tension forces and damped by the viscous forces. If the impact velocity is high enough, then the stretching will eventually cause the two thinnest sides of the ring to rupture. The rupturing of the ring can result in the formation of one or more "satellite droplets", as can be seen in Figure 14. If the impact velocity is not high enough, then the ring fully collapses and the newly formed droplet will oscillate with diminishing amplitude until a perfectly spherical droplet remains.

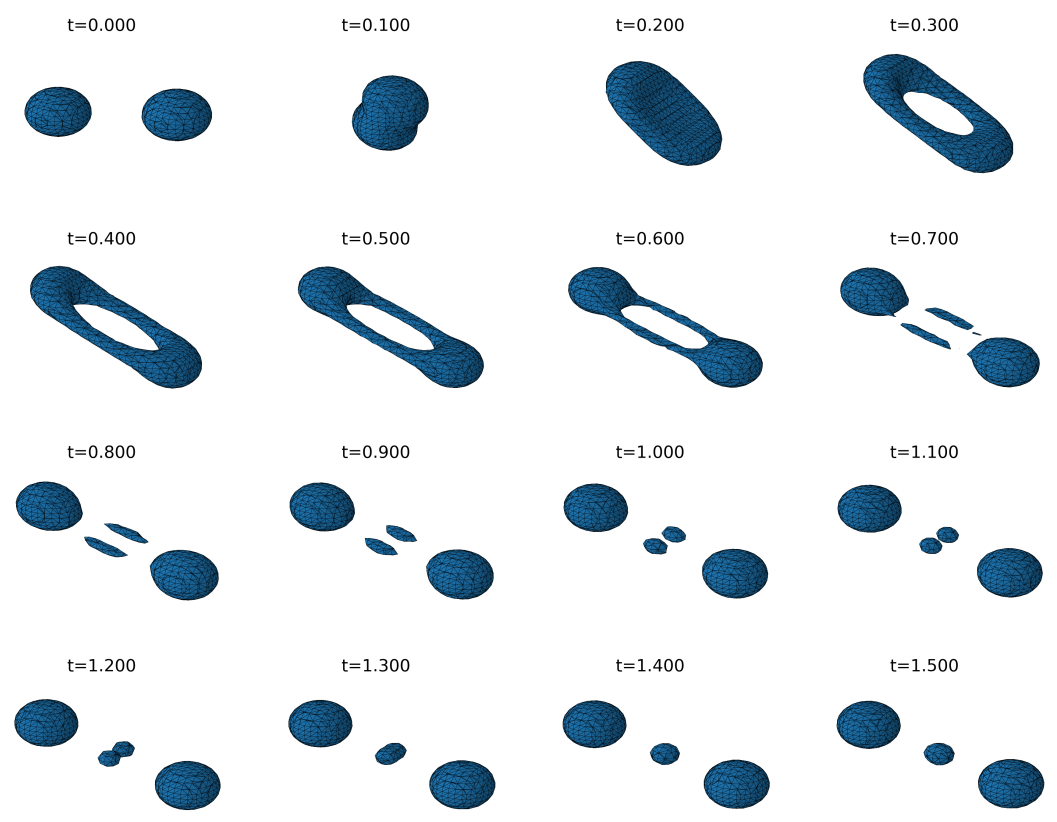

Figure 14: Example of an off-centre collision resulting in a stretching separation which forms a satellite droplet. $O h=0.258, W e_{c}=130, \mathscr{X}=0.5$

\subsection{Energy transfer and dissipation}

In this section the energy transfer and dissipation processes during the droplet collisions are studied in detail. As was expressed with equation 10, the total energy density equals the sum of the internal energy, kinetic energy and interfacial energy. The manner of conversion of kinetic energy into interfacial or internal energy is what dominates the outcome of the collisions. Analysis of this conversion process is the first step towards developing correlations that describe the boundaries between different collision outcomes. 
Because symmetry boundary conditions are imposed on all the boundaries of the domain, the total amount of energy in the domain should be constant throughout the simulations, apart from minor fluctuations due to roundoff errors. It has been verified that this is indeed the case for all simulations in the present study. It should also be noted that when other multiphase simulation methods are used to simulate droplet collisions, the total amount of energy is not guaranteed to be conserved. A decrease in the total amount of energy is observed during the collision process in the following studies: Nobari et al. [24], Rieber and Frohn [33], Sun et al. [41] and Rajkotwala et al. [31]. The loss in total energy can be as high as $20 \%$ and is usually caused by an equivalent loss in interfacial energy due to a sudden reduction of interfacial area. This reduction is caused by the inability of these simulation methods to completely capture all the interfacial dynamics during the collisions process. Diffuse Interface Methods do not suffer from this shortcoming because the interface region is fully resolved in a physically motivated manner.

The total amount of internal energy in the domain increases in all cases as a result of the dissipation of kinetic energy into heat due to viscous forces. Figure 15 shows histories of the total amount of internal, kinetic and surface energy for multiple collision regimes. The transfer of kinetic energy to surface energy at the beginning of the collision is easily understood from the stretching of the interface. For reflexive separation, the amount of surface energy at the end of the simulation is identical to the amount at the beginning of the simulation and all the kinetic energy has been converted into internal energy. For permanent coalescence, the amount of surface energy at the end of the simulation is lower than the amount at the beginning, since not only the kinetic energy but also a portion of the surface energy has been converted to internal energy. For a collision with a torus breakup it is the opposite: the amount of surface energy at the end of the simulation is higher than the amount at the beginning and a portion of kinetic energy has been converted to surface energy.
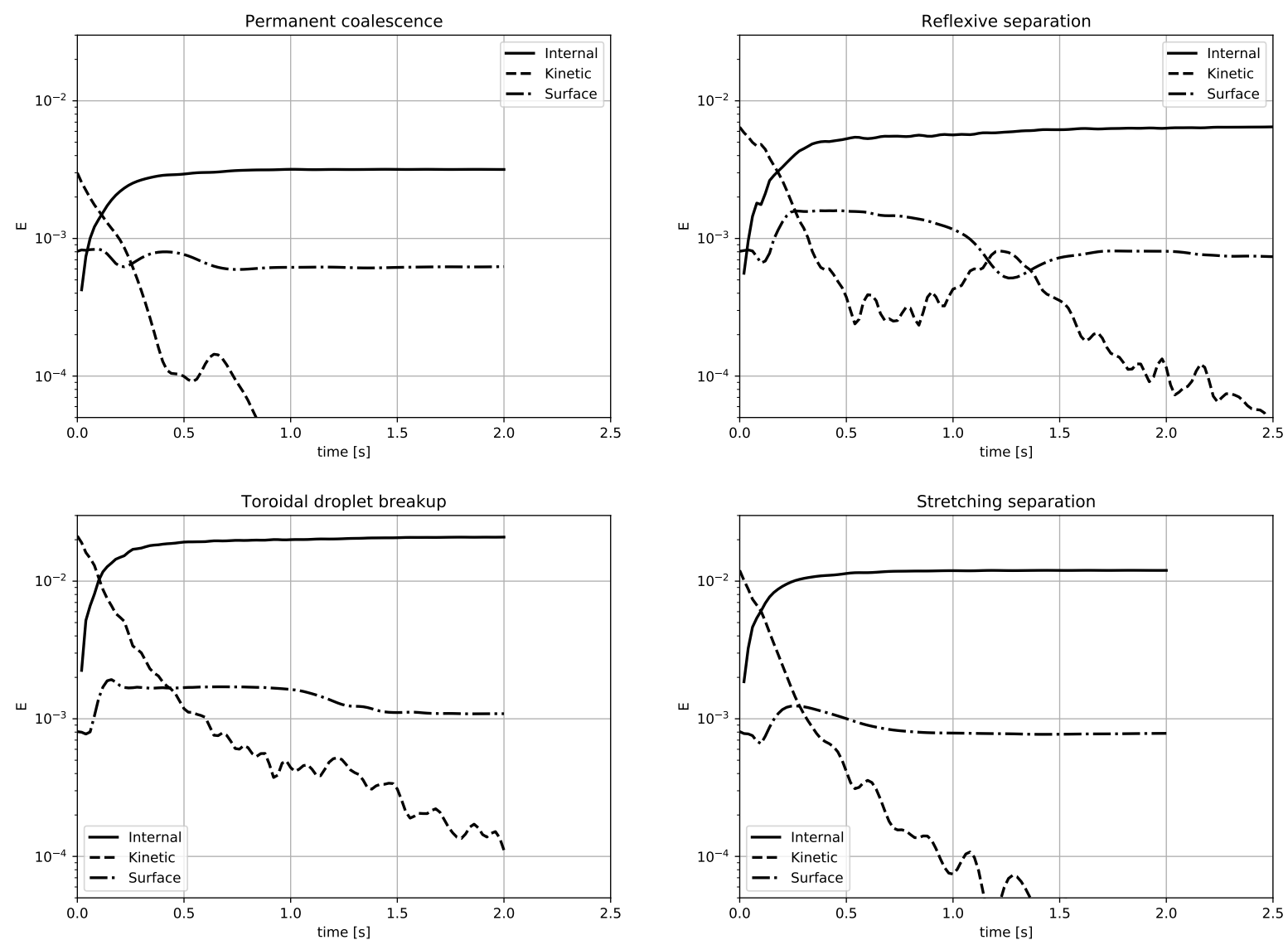

Figure 15: The total amount of internal, kinetic and surface energy during the simulation for the permanent coalescence, toroidal droplet breakup, reflexive separation and stretching separation 


\section{Conclusions}

A Diffuse Interface Model based on the Navier-Stokes-Korteweg equations has been developed and used to study the collision dynamics of droplets in three spatial dimensions and near the critical point of the fluid. No physical principles are violated or accounted for by empirical correlations, all the equations are derived from first principles i.e. conservation of mass/momentum/energy and fundamental thermodynamics. Simulation results showed the existence of various collision regimes including permanent coalescence, toroidal droplet breakup, reflexive separation and stretching separation. Additionally, the energy transfer and dissipation processes during the collision were studied in a level of detail that can only be obtained with DIM. The results highlight the importance of modeling liquid-vapor flows under non-isothermal conditions, since variations in the temperature field during the collision process are too large to be neglected and also influence the collision dynamics.

The regime of droplet bouncing was not observed in any of the simulations. In experimental investigations droplet bouncing is normally only observed when the droplets are surrounded by a gas of a different component at a sufficiently high pressure [30]. In the present study there is an important difference: the liquid droplets are surrounded by vapor comprised of the same molecules as the liquid, since the NSK equations can only be used for a single component fluid. Therefore, the vapor trapped between the droplets is able to condense and can be absorbed by the droplets. Is is therefore less likely that droplet bouncing will be be observed when the NSK equations are used.

It can be concluded that the Diffuse Interface Model gives detailed insight into droplet collision dynamics, which leads to a better understanding of the process. The results of simulations of droplet collisions with DIM can be used to either validate or improve coalescence models employed in sharp interface methods [18, 31]. It must be noted, however, that the temperature range in which DIM can be used is severely limited by a constraint on the grid spacing caused by the fact that the liquid-vapor interface becomes very thin when the temperature is far from the critical point. Additionally, the DIM described in this paper can only be used for liquid-vapor flows of a single species, whereas many industrial and geophysical processes with droplet collisions involve mixtures of fluids. Extending DIM to make it applicable to mixtures is the topic of further research. The use of mixing rules to extend the applicability of equations of state to mixtures is a well established practice in chemical engineering. The major difficulty is in the derivation of the Korteweg stress tensor and the inclusion of an additional convection-diffusion equation which accounts for non-uniformity in the composition of the mixture.

\section{Acknowledgments}

This work was sponsored by NWO Exacte en Natuurwetenschappen (Physical Sciences) for the use of supercomputer facilities, with financial support from the Nederlandse Organisatie voor Wetenschappelijk Onderzoek (Netherlands Organization for Scientific Research, NWO).

This work is part of the research programme Open Technologieprogramma with project number 13781, which is (partly) financed by the Netherlands Organisation for Scientific Research (NWO) Domain Applied and Engineering Sciences (TTW, previously Technology Foundation STW).

\section{References}

[1] Adalsteinsson, D., And Sethian, J. A. A fast level set method for propagating interfaces. Journal of computational physics 118, 2 (1995), 269-277.

[2] Aifantis, E. C., And Serrin, J. B. The mechanical theory of fluid interfaces and maxwell's rule. Journal of colloid and interface science 96, 2 (1983), 517-529.

[3] Ashgriz, N., And Poo, J. Coalescence and separation in binary collisions of liquid drops. Journal of Fluid Mechanics 221 (1990), 183-204.

[4] Brenn, G., And Kolobaric, V. Satellite droplet formation by unstable binary drop collisions. Physics of fluids 18, 8 (2006), 087101.

[5] Cahn, J. W., and Hilliard, J. E. Free energy of a nonuniform system. i. interfacial free energy. The Journal of chemical physics 28, 2 (1958), 258-267. 
[6] Carey, V. P. Liquid-vapor phase-change phenomena. Hemisphere, New York, NY (United States), 1992.

[7] Cockburn, B., And GaU, H. A model numerical scheme for the propagation of phase transitions in solids. SIAM Journal on Scientific Computing 17, 5 (1996), 1092-1121.

[8] Desmarais, J. Towards numerical simulation of phase-transitional flows. PhD thesis, Technische Universiteit Eindhoven, 2016.

[9] Desmarais, J., and Kuerten, J. Open boundary conditions for the diffuse interface model in 1-d. Journal of computational physics 263 (2014), 393-418.

[10] Dunn, J. E., And Serrin, J. On the thermomechanics of interstitial working. Archive for Rational Mechanics and Analysis 88, 2 (1985), 95-133.

[11] Dupuy, P. M., Lin, Y., Fernandino, M., Jakobsen, H. A., And Svendsen, H. F. Modelling of high pressure binary droplet collisions. Computers \& Mathematics with Applications 61, 12 (2011), 3564-3576.

[12] Estrade, J.-P., Carentz, H., Lavergne, G., and Biscos, Y. Experimental investigation of dynamic binary collision of ethanol droplets-a model for droplet coalescence and bouncing. International Journal of Heat and Fluid Flow 20, 5 (1999), 486-491.

[13] Finotello, G., Padding, J. T., Deen, N. G., Jongsma, A., Innings, F., And Kuipers, J. Effect of viscosity on droplet-droplet collisional interaction. Physics of Fluids 29, 6 (2017), 067102.

[14] Gotaas, C., Havelka, P., Jakobsen, H. A., Svendsen, H. F., Hase, M., Roth, N., and WeIGAND, B. Effect of viscosity on droplet-droplet collision outcome: Experimental study and numerical comparison. Physics of fluids 19, 10 (2007), 102106.

[15] Hirt, C. W., And Nichols, B. D. Volume of fluid (vof) method for the dynamics of free boundaries. Journal of computational physics 39, 1 (1981), 201-225.

[16] Jamet, D., Lebaigue, O., Coutris, N., and Delhaye, J. The second gradient method for the direct numerical simulation of liquid-vapor flows with phase change. Journal of Computational Physics 169, 2 (2001), 624-651.

[17] Krishnan, K., AND LOTH, E. Effects of gas and droplet characteristics on drop-drop collision outcome regimes. International Journal of Multiphase Flow 77 (2015), 171-186.

[18] Kwakkel, M., Breugem, W.-P., And Boersma, B. J. Extension of a clsvof method for dropletladen flows with a coalescence/breakup model. Journal of Computational Physics 253 (2013), 166-188.

[19] Lamorgese, A., AND Mauri, R. Diffuse-interface modeling of liquid-vapor phase separation in a van der waals fluid. Physics of Fluids 21, 4 (2009), 044107.

[20] Lewiner, T., Lopes, H., Vieira, A. W., and Tavares, G. Efficient implementation of marching cubes' cases with topological guarantees. Journal of graphics tools 8, 2 (2003), 1-15.

[21] Liu, J., Gomez, H., Evans, J. A., Hughes, T. J., and Landis, C. M. Functional entropy variables: a new methodology for deriving thermodynamically consistent algorithms for complex fluids, with particular reference to the isothermal navier-stokes-korteweg equations. Journal of Computational Physics 248 (2013), 47-86.

[22] Liu, J., Landis, C. M., Gomez, H., and Hughes, T. J. Liquid-vapor phase transition: thermomechanical theory, entropy stable numerical formulation, and boiling simulations. Computer Methods in Applied Mechanics and Engineering 297 (2015), 476-553.

[23] Meleán, Y., And Sigalotti, L. D. G. Coalescence of colliding van der waals liquid drops. International Journal of Heat and Mass Transfer 48, 19-20 (2005), 4041-4061.

[24] Nobari, M., Jan, Y.-J., And Tryggvason, G. Head-on collision of drops-a numerical investigation. Physics of Fluids 8, 1 (1996), 29-42.

[25] Onuki, A. Dynamic van der waals theory. Physical Review E 75, 3 (2007), 036304.

[26] Pairam, E., And Fernández-Nieves, A. Generation and stability of toroidal droplets in a viscous liquid. Physical review letters 102, 23 (2009), 234501.

[27] Papatzacos, P. Diffuse-interface models for two-phase flow. Physica Scripta 61, 3 (2000), 349. 
[28] Pecenko, A., Kuerten, J., And van der Geld, C. A diffuse-interface approach to two-phase isothermal flow of a van der waals fluid near the critical point. International Journal of Multiphase Flow 36,7 (2010), 558-569.

[29] Pecenko, A., Van Deurzen, L., Kuerten, J., And Van der Geld, C. Non-isothermal twophase flow with a diffuse-interface model. International Journal of Multiphase Flow 37, 2 (2011), 149-165.

[30] QIAN, J., AND LAW, C. Regimes of coalescence and separation in droplet collision. Journal of Fluid Mechanics 331 (1997), 59-80.

[31] Rajkotwala, A., Mirsandi, H., Peters, E., Baltussen, M., van der Geld, C., Kuerten, J., AND KuIPERS, J. Extension of local front reconstruction method with controlled coalescence model. Physics of Fluids 30, 2 (2018), 022102.

[32] Redlich, O., And Kwong, J. N. On the thermodynamics of solutions. v. an equation of state. fugacities of gaseous solutions. Chemical reviews 44, 1 (1949), 233-244.

[33] Rieber, M., And Frohn, A. Navier-stokes simulation of droplet collision dynamics. In Proc. 7th Int. Symp. On Comp. Fluid Dynamics, (ed. by Zhuang, FG) Beijing, China (1997), pp. 520-525.

[34] Roudbari, M. S., Simsek, G., van Brummelen, E. H., and van der Zee, K. G. Diffuseinterface two-phase flow models with different densities: a new quasi-incompressible form and a linear energy-stable method. arXiv:1603.06475 (2016).

[35] Shin, S., Yoon, I., And Juric, D. The local front reconstruction method for direct simulation of two-and three-dimensional multiphase flows. Journal of Computational Physics 230, 17 (2011), 66056646.

[36] Shu, C.-W., And Osher, S. Efficient implementation of essentially non-oscillatory shock-capturing schemes. Journal of Computational Physics 77, 2 (1988), 439-471.

[37] Shyu, G.-S., Hanif, N. S., Alvarado, J. F., Hall, K. R., And Eubank, P. T. Equal area rule methods for ternary systems. Industrial \& engineering chemistry research 34, 12 (1995), 4562-4570.

[38] Skowron, J., And Gould, A. General complex polynomial root solver and its further optimization for binary microlenses. arXiv:1203.1034 (2012).

[39] Slattery, J. C., Sagis, L., And OH, E.-S. Interfacial transport phenomena. Springer Science \& Business Media, 2007.

[40] Stryjek, R., And Vera, J. Prsv2: a cubic equation of state for accurate vapor-liquid equilibria calculations. The Canadian Journal of Chemical Engineering 64, 5 (1986), 820-826.

[41] Sun, K., Jia, M., And Wang, T. Numerical investigation of head-on droplet collision with lattice boltzmann method. International Journal of Heat and Mass Transfer 58, 1-2 (2013), 260-275.

[42] Sussman, M., And Puckett, E. G. A coupled level set and volume-of-fluid method for computing $3 \mathrm{~d}$ and axisymmetric incompressible two-phase flows. Journal of computational physics 162, 2 (2000), 301-337.

[43] Tian, L., Xu, Y., Kuerten, J., And van Der Vegt, J. J. A local discontinuous galerkin method for the (non)-isothermal navier-stokes-korteweg equations. Journal of computational physics 295 (2015), $685-714$.

[44] Van der WaAls, J. D. Over de Continuiteit van den Gas-en Vloeistoftoestand, vol. 1. Sijthoff, 1873.

[45] Wemhoff, A. P. Dependence of the equation of state in surface tension prediction by the theory of capillarity. In ASME 2009 International Mechanical Engineering Congress and Exposition (2009), American Society of Mechanical Engineers, pp. 1999-2005.

[46] Willis, K., And ORme, M. Binary droplet collisions in a vacuum environment: an experimental investigation of the role of viscosity. Experiments in fluids 34, 1 (2003), 28-41.

[47] Yue, P., Feng, J. J., Liu, C., And Shen, J. A diffuse-interface method for simulating two-phase flows of complex fluids. Journal of Fluid Mechanics 515 (2004), 293-317. 